ARTICLE

DOI: $10.1038 / \mathrm{s} 41467-018-07656-2$

\title{
Layer-edge device of two-dimensional hybrid perovskites
}

Bin Cheng (1) ${ }^{1}$, Ting-You Li ${ }^{1}$, Pai-Chun Wei ${ }^{1}$, Jun Yin (D) ${ }^{2}$, Kang-Ting Ho ${ }^{1}$, José Ramón Durán Retamal ${ }^{1}$, Omar F. Mohammed ${ }^{2} \& \mathrm{Jr}-\mathrm{Hau} \mathrm{He}^{1}$

Two dimensional layered organic-inorganic hybrid perovskites (2D perovskites) are potential candidates for next generation photovoltaic device. Especially, the out-of-plane surface perpendicular to the superlattice plane of 2D perovskites (layer-edge surface) has presented several exotic behaviors, such as layer-edge states which are found to be crucial for improving the efficiency of 2D perovskite solar cells. However, fundamental research on transport properties of layer-edge surface is still absent. In this report, we observe the electronic and opto-electronic behavior in layer-edge device of 2D perovskites. The dark and photo currents are demonstrated to strongly depend on the crystallographic orientation in layer-edge device, and such anisotropic properties, together with photo response, are related to the thickness of inorganic layers. Finally, due to the abundant hydroxyl groups, water molecules are easy to condense on the layer-edge surface, and the conductance is extremely sensitive to the humidity environment, indicating a potential application of humidity sensor.

\footnotetext{
${ }^{1}$ Computer, Electrical, and Mathematical Sciences and Engineering Division, King Abdullah University of Science and Technology, Thuwal 23955-6900, Saudi Arabia. ${ }^{2}$ Physical Sciences and Engineering Division, King Abdullah University of Science and Technology, Thuwal 23955-6900, Saudi Arabia. These authors contributed equally: Bin Cheng, Ting-You Li. Correspondence and requests for materials should be addressed to J.-H.H. (email: jrhau.he@kaust.edu.sa)
} 
O rganic-inorganic hybrid perovskites $\mathrm{MAPbX}_{3}(\mathrm{MA}=$ $\mathrm{CH}_{3} \mathrm{NH}_{3}, \mathrm{X}=\mathrm{Cl}, \mathrm{Br}, \mathrm{I} ; 3 \mathrm{D}$ perovskites for brief) have achieved a power conversion efficiency (PCE) of over $20 \%^{1-5}$ in photovoltaic solar cells and also are promising materials for fabricating light-emitting diodes ${ }^{6,7}$ and lasers ${ }^{8,9}$. Just like conventional semiconductor device ${ }^{10,11}$, the surfaces and interfaces have a key role in the electronic and opto-electronic performance in the hybrid perovskites based photovoltaic devices $^{12,13}$. Especially in the two-dimensional-layeredorganic-inorganic hybrid perovskites (2D perovskites), the outof-plane surface perpendicular to the superlattice plane, which is named as layer-edge surface, has unique organic-inorganic staggered superlattice pattern, as a straightforward result of multiple quantum well structures. Because of the parasitic layeredge states, such exotic layer-edge surface has a crucial role in dissociating the electron-hole pairs in the excitons into the free carriers and extend the lifetime of those carriers, leading to a largely improved efficiency in 2D perovskite based solar cells ${ }^{14,15}$. Moreover, a generic elastic model was supposed, demonstrating that the layer thickness-dependent electronic band structure of layer-edge surface in $2 \mathrm{D}$ perovskites comes from relaxation of mechanical strain in the mismatched interface ${ }^{16}$. Those impressive results presented fancy properties of the layer-edge surface in $2 \mathrm{D}$ perovskites, but several other important fundamental researches are still required, especially the electronic transport properties which is one of the key factors to determine the performance of opto-electronic devices.

Here, we succeed to grow centimeter-wide and millimeterthick single crystalline $2 \mathrm{D}$ perovskites, and fabricate the metal-semiconductor-metal devices on the layer-edge surface in the direction parallel to the $\mathrm{Pb}-\mathrm{I}$ plane (defined as layer-edge $0^{\circ}$ device) and perpendicular to the $\mathrm{Pb}-\mathrm{I}$ plane (defined as layeredge $90^{\circ}$ device), respectively. Transport measurements are performed in these layer-edge devices of $2 \mathrm{D}$ perovskites, showing highly anisotropic electrical and opto-electronic conductance. Moreover, as the thickness of the Pb-I layer increases, the anisotropy is less pronounced, and the rise time and recover time of photo response decrease. Finally, the layer-edge device is demonstrated to be extremely sensitive to environmental humidity, due to the abundant hydroxyl groups which can help water condensation.

\section{Results}

Sample preparation and layer-edge device fabrication. First, in order to fabricate the layer-edge device, 2D perovskite single crystal with large enough layer-edge surface is required. However, in the commonly studied 2D perovskites, because the Van der Waals interaction between the organic layers is quite weak, an extreme preference of growing along the $\mathrm{Pb}-\mathrm{I}$ plane leads to a limited thickness of single crystals. In order to increase the growth preference perpendicular to the $\mathrm{Pb}$-I plane, we used large polar organics, $\mathrm{HOC}_{2} \mathrm{H}_{4} \mathrm{NH}_{3}{ }^{+}$(EA), to form the organic layers so that dipole-dipole force can largely enhance the interaction between organic layers ${ }^{17,18}$. By a cooling method (see Methods), centimeter-wide and millimeter-thick single crystals of $\left(\mathrm{HOC}_{2} \mathrm{H}_{4} \mathrm{NH}_{3}\right)_{2} \mathrm{PbI}_{4}$ were successfully synthesized (Supplementary Figure 1). The lattice structure of the 2D_EA perovskites is confirmed by single-crystal X-ray diffraction (SCXRD), and the layer-edge surfaces are determined (see Methods and Supplementary Figure 2).

For the purpose of investigating the electronic and optoelectronic transport properties of layer-edge surface of $2 \mathrm{D}$ perovskites, both layer-edge $0^{\circ}$ and layer-edge $90^{\circ}$ metal-semiconductor-metal devices are fabricated by depositing $\mathrm{Au}$ electrodes with the same active area and deposition conditions (schematically shown in Fig. 1a). The top view of the conducting channel of layer-edge device is schematically shown in Fig. 1b. The optical image of the layer-edge surface device is shown in Fig. 1c, in which the electrode pairs of 1 to 3 and 2 to 4 are layeredge $0^{\circ}$ device, while the electrode pairs of 1 to 2 and 3 to 4 indicate layer-edge $90^{\circ}$ device. Device on the in-plane surface was also fabricated for comparative experiments (Supplementary Figure 3). From the AFM data, we can get that the roughness of the layer-edge surface and in-plane surface is about $5 \mathrm{~nm}$ (Fig. 1d) and $1 \mathrm{~nm}$ (Supplementary Figure 4). The contact resistance is much smaller than the intrinsic resistance of sample, so the contact resistance in our transport measurements is neglected (see Methods). Moreover, the corrections according to the geometry difference of channels in the layer-edge $90^{\circ}$ and $0^{\circ}$ devices are considered (see Methods).

$I-V$ characterization of layer-edge device. The $I-V$ curve (Fig. 2a) shows that the dark current in layer-edge $0^{\circ}$ device is about two-orders larger than that in layer-edge $90^{\circ}$ device, while the photo current of layer-edge $0^{\circ}$ and layer-edge $90^{\circ}$ device show about one-order difference. In the photo responsivity measurements, we fixed the bias at 3 volts and measured the photo responsivity over a wide range of incident light wavelength from 360 to $600 \mathrm{~nm}$ (Fig. 2b), indicating that layer-edge $0^{\circ}$ and $90^{\circ}$ devices have the similar wavelength dependence, and the largest photo responsivity was found at $550 \mathrm{~nm}$ wavelength. Meanwhile, the magnitude of photo responsivities in the layer-edge $0^{\circ}$ device is close to that in the in-plane device (Supplementary Figure 5), and about one-order larger than that in the layer-edge $90^{\circ}$ device (Fig. 2b). Such anisotropy of transport properties is similar as the anisotropy observed in the excitonic response in $2 \mathrm{D}$ perovskites $^{19}$, indicating that carrier transport mainly occurs along $\mathrm{Pb}-\mathrm{I}-\mathrm{Pb}$ inorganic pathways while is blocked between different inorganic layers owing to confinement by the organic layers, as shown schematically in Fig. 1a. Our study illustrates that in 2D perovskite thin film device, random orientation of crystalline domains will create massive obstacles for carrier transport, hindering the photovoltaic application and fundamental research.

The illumination power density dependent photo current was measured at 3 volts bias voltage and the $550 \mathrm{~nm}$ wavelength of incident light, so that the photo responsivity has maximum value. As shown in Fig. 2c, the photo current $I_{\mathrm{p}}$ and photoexcitation density $D$ has the power law relations $I_{\mathrm{p}} \propto D^{m}$. Both layer-edge $0^{\circ}$ and layer-edge $90^{\circ}$ devices show two distinct power law regions: when the photoexcitation density is low, the trap-assisted recombination dominates with $m=\frac{2}{3}$; as the photoexcitation density is increased, the bimolecular recombination becomes the dominating mechanism, leading to $m=\frac{1}{3}$. The decrease of $m$ compared to the idea value of $1 / 2$ and 1 for the two recombination regimes in $2 \mathrm{D}$ perovskite in-plane device has been reported by other groups ${ }^{20}$, where two possible reasons were discussed: (1) exciton-exciton interactions which is largely enhanced in low-dimensional systems, and (2) limited collection of photo-generated charges blocked by insulating organic layers. In the layer-edge device, the many-body interactions still have a key role, while the blockade of charge collection by insulating layers does not exist. Another possible reason of decreased $m$ in layer-edge device is proposed here: when the charge carriers are generated at surface, the carriers can diffuse into the bulk before they recombine; as the incident light density increases, the carrier diffusion is suppressed due to carrier recombination, leading to a decrease of carrier lifetime, and finally result in an effective desensitization of photo conductivity ${ }^{21}$.

We also succeeded to grow 2D perovskites with different thickness of inorganic layers. Here we introduce the chemical 
a



b

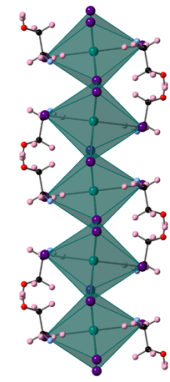

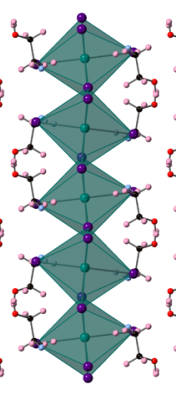





C


Fig. 1 Layer-edge device. a Schematic diagram of layer-edge surface of $2 \mathrm{D}$ perovskite $\left(\mathrm{HOC}_{2} \mathrm{H}_{4} \mathrm{NH}_{3}\right)_{2} \mathrm{Pbl}_{4}$. Blue arrow and red arrow between electrodes indicate the direction of layer-edge $90^{\circ}$ and $0^{\circ}$, respectively. b Top view of layer-edge surface. c Optical image of layer-edge surface device. The scale bar is $400 \mu \mathrm{m}$. d AFM image of layer-edge surface
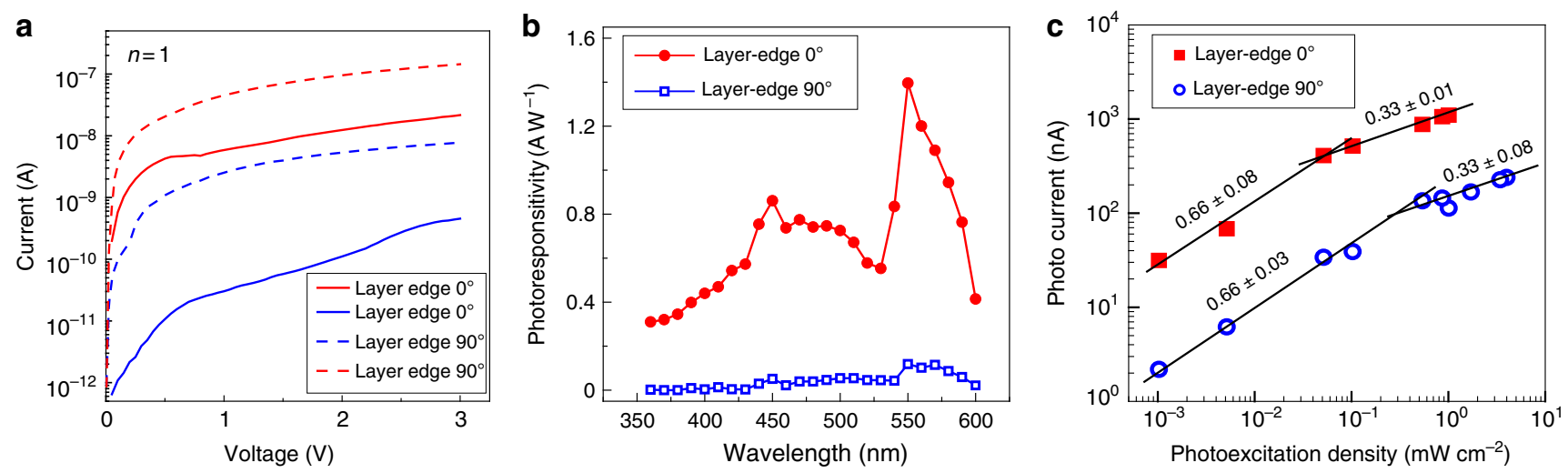

Fig. 2 Transport properties of layer-edge device with $n=1$. a $l-V$ curve in dark and photo environment under white light with the power of $1 \mathrm{~mW} \mathrm{~cm}^{-2}$. $I_{\mathrm{d}}$ is dark current and $I_{\mathrm{p}}$ is photo current for layer-edge $0^{\circ}$ and $90^{\circ}$ devices. b Photo responsivity vs wavelength. The light power density is $10 \mathrm{~mW} \mathrm{~cm}^{-2}$. $\mathbf{c}$ Photo current vs photoexcitation density in layer-edge $0^{\circ}$ and $90^{\circ}$ devices. The bias voltage is fixed at $3 \mathrm{~V}$ in $\mathbf{b}$ and $\mathbf{c}$

formula $\mathrm{EA}_{2} \mathrm{MA}_{n-1} \mathrm{PbI}_{3 n+1}$, where $\mathrm{EA}=\mathrm{HOC}_{2} \mathrm{H}_{4} \mathrm{NH}_{3}{ }^{+}$and $\mathrm{MA}=\mathrm{CH}_{3} \mathrm{NH}_{3}{ }^{+}$, and $n$ represents the number of inorganic layers between two ammonium organic layers consisting of EA. The single-crystal structure of $\mathrm{EA}_{2} \mathrm{MA}_{n-1} \mathrm{PbI}_{3 n+1}$ for $n=1,2$, and 3 are determined by Single Crystal XRD, and the positions of missing organic groups are decided by optimization at GGA/PBE level of theory, as shown in Fig. 3a. Basically, enlarging the thickness of the semiconductor layer in a quantum well structure will decrease the quantum confinement ${ }^{22-24}$. In order to investigate the effect of quantum confinement on the transport properties of layer-edge surface device, the $I-V$ curve under dark and photo environment for $2 \mathrm{D}$ perovskites with $n=2$ and 3 were measured, as shown in Fig. $3 b$ and c. Here we define the anisotropy of conductance and photo conductance as the ratio of current with bias equals to 3 volts in layer-edge $0^{\circ}$ device and $90^{\circ}$ device. As shown in Fig. $3 \mathrm{~d}$, The anisotropy is decreased as $n$ is increased; when $n \rightarrow \infty$ the case of $3 \mathrm{D}$ perovskite is approached, and the electronic anisotropy is minimized and equals to one.

Generally, layer-edge $0^{\circ}$ and layer-edge $90^{\circ}$ devices have different transport mechanism. In layer-edge $0^{\circ}$ device, the carrier transport mainly happens within the individual semiconductor layers; when the external electric field is applied parallel to the semiconductor layers, the carriers are drifted until scattered by charge impurities or phonons. So the transport in layer-edge $0^{\circ}$ device can perfectly described by Drude model, in which the mobility is determined by effective mass and scattering relaxation time. On the other hand, in layer-edge $90^{\circ}$ device, the dominating process of transport is tunnelling through organic 
a


Fig. 3 Thickness-dependent electronic/opto-electronic properties. a Schematic image of 2D perovskites with $n=1,2,3$, and $\infty$. Dark and photo current of $2 \mathrm{D}$ perovskite with $\mathbf{b} n=2$ and $\mathbf{c} n=3$. d Anisotropy of conductance and photo conductance with different $n$
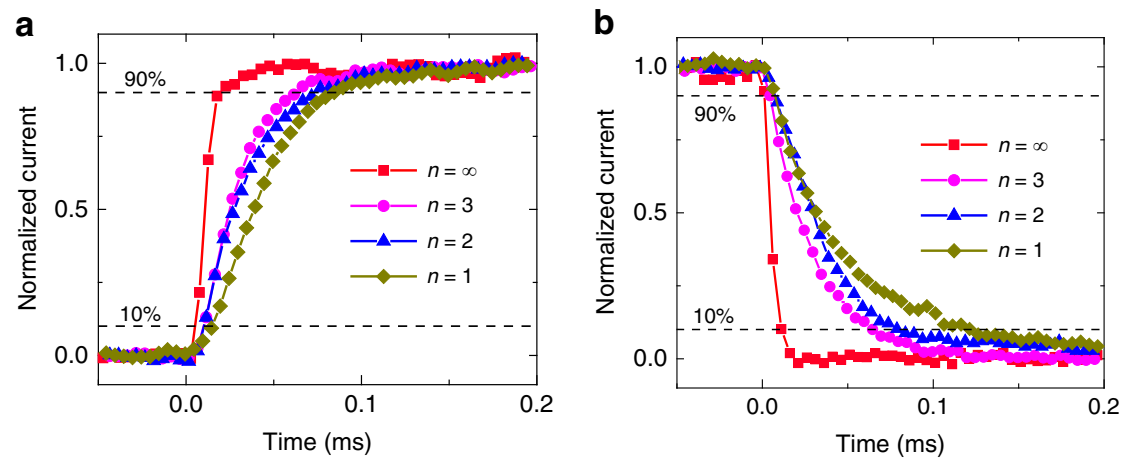

Fig. 4 Thickness-dependent photo response. a Photo response and $\mathbf{b}$ recovery of normalized current in 2D perovskite with $n=1,2$, and 3 and $3 D$ perovskite

\begin{tabular}{|c|c|c|c|c|}
\hline & $n=1$ & $n=\mathbf{2}$ & $n=\mathbf{3}$ & $\boldsymbol{n}=\boldsymbol{\infty}$ \\
\hline Rise time $(\mu \mathrm{s})$ & 69.8 & 63.9 & 54.4 & 15.0 \\
\hline Recover time $(\mu \mathrm{s})$ & 106.9 & 74.2 & 50.0 & 10.3 \\
\hline
\end{tabular}

barriers, and Drude model is invalid. Actually, when we estimated the effective mass from the DFT calculated band structures (Supplementary Figure 6), the effective masses along semiconductor plane (the situation in layer-edge $0^{\circ}$ device) are similar for $n=1,2$, and 3 ; While the effective mass perpendicular to the semiconductor plane is smallest for $n=1$, and close to infinite for $n=2$ and 3, as shown in Supplementary Table 1. Those effective masses cannot explain the $n$ dependent anisotropy of conductivity in the sense of Drude model, confirming that Drude model cannot be used for layer-edge $90^{\circ}$ device.
Photo response of layer-edge device. To study the photoresponse dynamics in layer-edge surface devices, we measured the time-dependent photo current of $2 \mathrm{D}$ perovskites $(n=1,2,3)$ and $3 \mathrm{D}$ perovskites. The timescales of photo-response dynamics measurement are quite larger than the carrier of lifetime in regular time-resolved photoluminescence measurement, since the external electric field will dissemble the electron-hole pairs in the excitons, and effectively extend the carrier lifetime ${ }^{25}$. The response time $\tau$ is defined by the duration of photo current changing from 10 to $90 \%$ of the maximum and vice versa. The rise and recover current curves are shown in Fig. $4 \mathrm{a}$ and b, respectively, and the response time $\tau$ is decreased with the increasing layer number $n$ and reaches the minimum value as $n=\infty$ (3D perovskites), as shown in Table 1 . The tendency of decrescent $\tau$ is highly associated with the quantum confinement effect, originated from the organic-inorganic-organic sandwich structures in $2 \mathrm{D}$ perovskites: as $n$ increases, the quantum confinement effect is weakened, resulting in the increase of carrier 

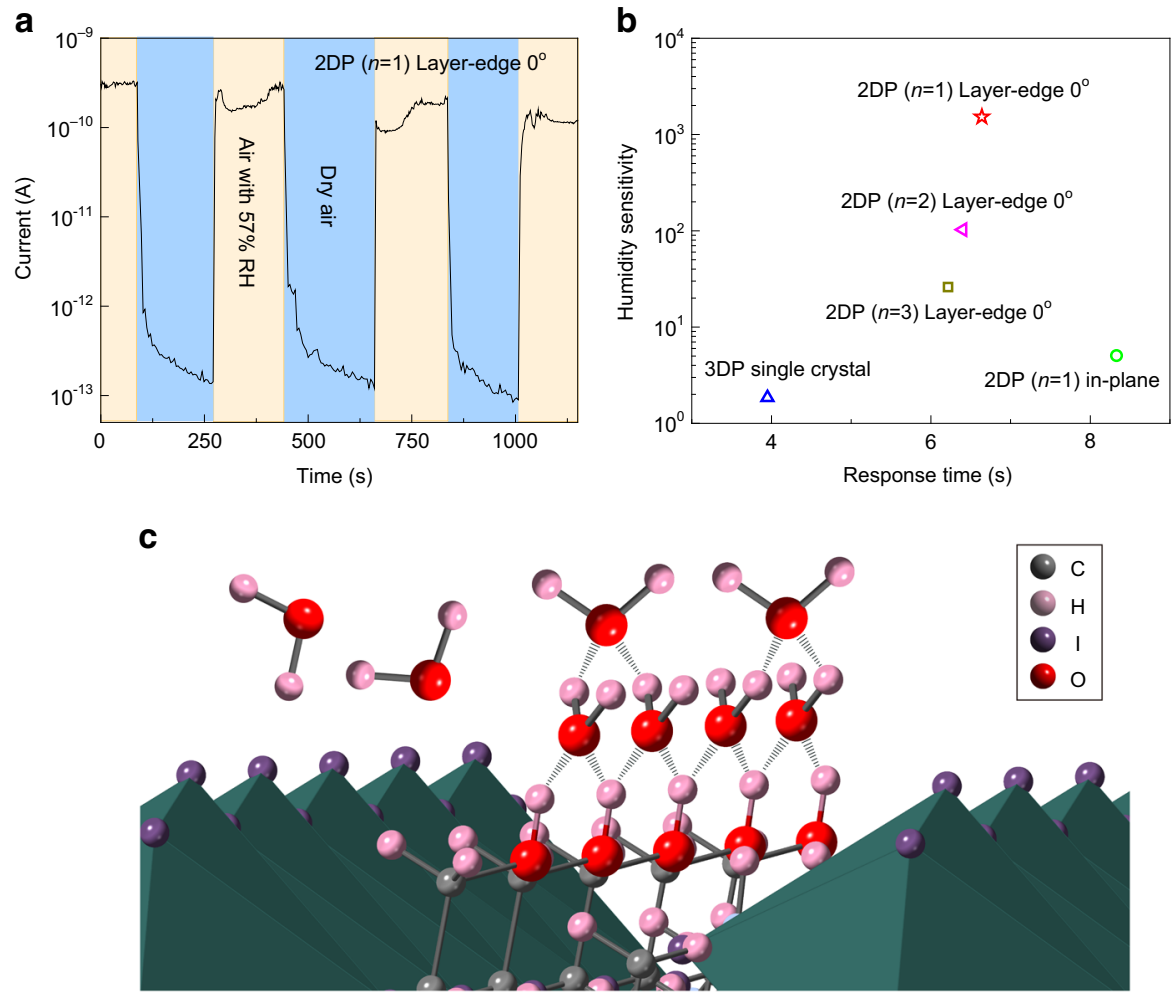

Fig. 5 Humidity sensitivity. a Dark current in dry air and with $57 \%$ humidity air in 2D perovskite layer-edge $0^{\circ}$ device. b Humidity sensitivity and response time for devices on layer-edge $0^{\circ}$ surface with different $n$, in-plane surface in 2D perovskites with $n=1$, and (110) surface in 3D perovskites. c Schematic diagram of water absorption in layer-edge surface of 2D perovskites

mobility 22 that shortens the drift time of photo-generated carriers to the electrodes, and finally making the $\tau$ decrease.

Humidity sensing of layer-edge device. Since the surface of perovskite device is significantly influenced by the environment gases and humidity, the transport behavior, which mainly occurs near the surface of the single-crystal device, are quite sensitive to the humidity when the device is operated in the air. In $3 \mathrm{D}$ perovskites, the sensitivity of humidity have been studied in the single crystal ${ }^{26}$ and thin film ${ }^{27}$. However, the result in $2 \mathrm{D}$ perovskites is expected to be quite different because of its layered structure and different organic groups adopted, especially in the layer-edge device some abnormal humidity sensitivity may show up. As shown in Fig. 5a, the currents of layer-edge $0^{\circ}$ devices (with $n=1$ ) in dry air and $57 \%$ relative humidity (RH) air are measured (see Methods), showing reversible three-ordermagnitude change, and response time to the water absorption as fast as approximately $6.6 \mathrm{~s}$. Here the response time is defined as the time required to change the current amplitude at dry air to the $90 \%$ maximum at $57 \% \mathrm{RH}$. The humidity response for layeredge device with $n=2$ and 3 (Supplementary Figure 7), layeredge device $90^{\circ}$ and in-plane device in $2 \mathrm{D}$ perovskite with $n=1$, and (110) surface device in 3D perovskite were also measured (Supplementary Figure 8). We estimate the performance of humidity sensing for each perovskite device by the humidity sensitivity (dividing the current in $57 \% \mathrm{RH}$ by the current in dry air), and the response time, as shown in Fig. 5b. The 2D perovskite layer-edge $0^{\circ}$ device with $n=1$ presents the best humidity sensitivity, and relative outstanding response time. Especially compared to the best humidity sensor based on hybrid perovskite so $\mathrm{far}^{28}$, the performance of humidity sensitivity and response time in $2 \mathrm{D}$ perovskite layer-edge device with $n=1$ are much more excellent. As $n$ increases, the humidity sensitivity of layer- edge device is decreased. The reason is that the ratio of organic layers to the inorganic layers will decrease as $n$ increases, which means that the density of hydroxyl groups on the layer-edge surface decreases, and the ability to absorb water decreases as well.

Now we try to explain such marked high humidity sensitivity of layer-edge device. First of all, the layer-edge surface of $2 \mathrm{D}$ perovskite is very rough as shown in the AFM image (Fig. 1d), especially compared to the in-plane surface (after mechanical exfoliation) in which the AFM shows atomic flat surface with much less defects (Supplementary Figure 4). Such structural defects in layer-edge surface can trap a lot of water molecules, which is proved by the large difference between the humidity sensitivity of layer-edge surface device and in-plane surface device (Supplementary Figure 8b). On the other hand, the layer-edge surface has abundant hydroxyl groups, and is easy to absorb the water in the air (as shown schematically in Fig. 5c). This mechanism can be verified when we look at the speed of current recovery when the device is put back to dry air from $57 \%$ humidity air. The current recovery speed in $2 \mathrm{D}$ perovskite with hydroxyl groups (Fig. 5a) is much slower than that in 2D perovskite $\left(\mathrm{C}_{6} \mathrm{H}_{5}\left(\mathrm{CH}_{2}\right)_{2} \mathrm{NH}_{3}\right)_{2} \mathrm{PbI}_{4}$ (2D_PEA perovskites) in which hydroxyl groups are absent (Supplementary Figure 9), indicating a process of desorbing water from hydroxyl groups. The type of moving charges in the layer-edge surface device in humidity environment is still unknown. The electronic carriers might contribute to the high humidity sensitivity since the doping water may change the surface energy band structures $^{29}$, or increase the carrier (electron or hole) concentration. On the other hand, when the water is condensed at the layer-edge surface, the water molecules may hydrolyze the perovskite lattices ${ }^{30}$, and create huge 
amounts of moving ions. Finally, protons can also transfer between the water molecules, which is called Grotthuss mechanism $^{31,32}$.

\section{Discussion}

In summary, we succeed to fabricate the layer-edge surface device of 2D hybrid perovskites with different inorganic layer thickness, and investigate their transport properties. Strong anisotropic electronic/opto-electronic properties are demonstrated, caused by the organic/inorganic layer staggered multiple quantum well structures. Such anisotropy is decreased as the thickness of inorganic layer increases. The existence of large anisotropy of electronic transport in 2D perovskites indicates the importance of the choice of crystalline orientation in the layer-edge device. We also obtain extremely high humidity sensitivity in the layer-edge device due to the abundant hydroxyl groups. The humidity sensing properties of layer-edge device in $2 \mathrm{D}$ perovskite strongly depend on the organic groups adopted, indicating urgent requirement of research on $2 \mathrm{D}$ perovskites with different organic layers in the future. Our study has revealed the unusual transport properties of layer-edge surface, and indicated potential applications of humidity sensor based on $2 \mathrm{D}$ perovskite single crystals.

\section{Methods}

Synthesis of perovskite single crystals. $2 \mathrm{D}$ perovskites $\left(\mathrm{HOC}_{2} \mathrm{H}_{4} \mathrm{NH}_{3}\right)$ single crystals are grown using a cooling method. First, $1.6 \mathrm{~g} \mathrm{PbI}_{2}$ is put in $16 \mathrm{ml}$ of $57 \%$ $\mathrm{HI}$ solution in a sample vial, and dissolved by sonication at room temperature. Thereafter, $0.8 \mathrm{ml} \mathrm{HOC} \mathrm{H}_{4} \mathrm{NH}_{2}$ was add and the precipitate. The solution is then cooled down to $4{ }^{\circ} \mathrm{C}$. After one week, the crystal was taken out and put in a fresh prepared solution. Such process was repeated for 5 weeks.

In order to grow $n=2$ perovskites, we prepare $16 \mathrm{ml} \mathrm{HI} \mathrm{(57} \mathrm{wt.} \mathrm{\% )} \mathrm{and} \mathrm{put} \mathrm{in}$ $5.5 \mathrm{~g} \mathrm{PbI}_{2}$ powder. After the powder dissolved, add $0.4 \mathrm{ml} \mathrm{MA}$ solvent and $0.8 \mathrm{ml}$ EA solvent, then heat it to $90^{\circ} \mathrm{C}$. After $1 \mathrm{~h}$, cool the solvent to $60^{\circ} \mathrm{C}$ and keep it for 2 days, then cool it down to room temperature. Then large size single crystal will be synthesized in a few days. For $n=3$ perovskite single crystal, we prepare $12 \mathrm{ml} \mathrm{HI}$ solution, we need to add $5 \mathrm{~g} \mathrm{PbI}_{2}$ powder, $0.4 \mathrm{ml} \mathrm{MA}$ and $0.8 \mathrm{ml} \mathrm{EA}$. Then the heating and cooling process is the same as $n=2$ perovskites.

Single crystal XRD of 2D perovskite. Single-crystal intensity data were collected using a Bruker APEX DUO with Mo $K_{\alpha}$ radiation (wavelength $0.71073 \AA$ ) at $100 \mathrm{~K}$. The cif file is upload to CCDC database, as shown in Data availability. The crystallographic plane of the large single crystal was probed using a Bruker D8 Venture SCXRD $\mathrm{Cu}$ target.

Determine the surface of single crystal. As shown in Supplementary Figure 1, all the single crystals have a shape of thin plate, and the largest surface is defined as inplane surface. The in-plane surface is parallel to the quantum well superlattice plane, which is proved by the XRD on the in-plane surface, as shown in Supplementary Figure 2a. The layer-edge surface is perpendicular to the in-plane surface as shown in Supplementary Figure $2 \mathrm{~b}$, so we can confirm that the layer-edge surface is perpendicular to the superlattice plane.

Electrical and opto-electronic characterization/optical testing. Electrical properties were measured using a Keithley $4200-$ SCS parameter analyzer with a Keithley 4210-PA. A Newport 1000 W Xenon Lamp 66921 light source with a Newport monochromator was used for the photo-responsivity measurements. To confirm the size and uniform intensity distribution of the incident light spot, the optical profile was measured by a beam profiling camera (Ophir Spiricon silicon CCD camera SP620U) and the power at each wavelength was measured using a calibrated photodiode (Newport 818-UV). The photo responsivity was calculated using the following formula:

$$
\text { Photoresponsivity }=\left(I_{\mathrm{p}}-I_{\mathrm{d}}\right) / D
$$

where $I_{\mathrm{p}}$ and $I_{\mathrm{d}}$ are the currents under photoexcitation and in the dark, respectively, and $D$ is the illumination power in active channel area in the device.

The setup of photo-response measurement is shown in Supplementary Figure 10.

The setup of current measurement with various atmosphere ambient is shown in Supplementary Figure 11
Contact resistance. As shown in Supplementary Figure 12a, we apply a voltage bias $\left(V_{\mathrm{b}}\right)$ on the electrode 2 to 3 , and measure the current $(I)$. The measured resistance between electrodes 2 to 3 include the intrinsic resistance of crystal $\left(R_{\mathrm{i}}\right)$ and contact resistance $\left(R_{\mathrm{c}}\right)$ between the crystal and the electrodes, i.e., $R_{\mathrm{i}}+R_{\mathrm{c}}=$ $V_{\mathrm{b}} / I_{2-3}$.

Then, as shown in Supplementary Figure 12b, the voltage bias is applied between the electrode 1 to 4 , and we measure the current $I$ and the voltage drop $V_{\mathrm{b}}$ between electrodes 2 to 3 at the same time. Then we can exclude the contact resistance and only measure the intrinsic resistance between electrode 2 to $3: R_{\mathrm{i}}=$ $V_{2-3} / I_{1-4}$

Finally, we find that $V_{\mathrm{b}} / I_{2-3} \approx V_{2-3} / I_{1-4}$, so $R_{\mathrm{i}}+R_{\mathrm{c}} \approx R_{\mathrm{i}}$. As a result, we can neglect the contact resistance for the $I-V$ measurement.

Different device geometry. Also, the channel length between two electrodes is kept at $50 \mu \mathrm{m}$; while we try our best to make the channel width $l$ close to $500 \mu \mathrm{m}$. But sometimes, the geometry may be different for different devices. In order to eliminate the effect of different channel geometry when we calculate the anisotropy of resistance, we do some corrections:

$$
\text { Anisotropy }=\left(I_{\text {layer-edge } 0^{\circ}} / I_{\text {layer-edge } 90^{\circ}}\right) \times\left(I_{\text {layer-edge } 90^{\circ}} / I_{\text {layer-edge } 0^{\circ}}\right)
$$

Where $I$ is the current, and $l$ is the channel width

\section{Data availability}

The data that support the findings of this study are available from the corresponding author upon reasonable request. The X-ray crystallographic coordinates for the structure of $2 \mathrm{D}$ perovskites has been deposited at the Cambridge Crystallographic Data Centre (CCDC), under deposition number 1869720 for $\left(\mathrm{HOCH}_{2} \mathrm{CH}_{2} \mathrm{NH}_{3}\right)_{2} \mathrm{PbI}_{4}$. These data can be obtained free of charge from The Cambridge Crystallographic Data Centre via www.ccdc.cam.ac.uk/data_request/cif.

Received: 27 June 2018 Accepted: 16 November 2018

Published online: 05 December 2018

\section{References}

1. Lee, M. M., Teuscher, J., Miyasaka, T., Murakami, T. N. \& Snaith, H. J. Efficient hybrid solar cells based on meso-superstructured organometal halide perovskites. Science 338, 643-647 (2012).

2. Jeon, N. J. et al. Compositional engineering of perovskite materials for highperformance solar cells. Nature 517, 476-480 (2015).

3. Burschka, J. et al. Sequential deposition as a route to high-performance perovskite-sensitized solar cells. Nature 499, 316-319 (2013).

4. Zhou, H. et al. Interface engineering of highly efficient perovskite solar cells. Science 345, 542-546 (2014)

5. Yang, W. S. et al. High-performance photovoltaic perovskite layers fabricated through intramolecular exchange. Science 348, 1234-1237 (2015).

6. Cho, H. et al. Overcoming the electroluminescence efficiency limitations of perovskite light-emitting diodes. Science 350, 1222-1225 (2015).

7. Chin, X. Y. et al. Lead iodide perovskite light-emitting field-effect transistor. Nat. Commun. 6, 7383 (2015).

8. Xing, G. et al. Low-temperature solution-processed wavelength-tunable perovskites for lasing. Nat. Mater. 13, 476-480 (2014).

9. Deschler, F. et al. High photoluminescence efficiency and optically pumped lasing in solution-processed mixed halide perovskite semiconductors. J. Phys. Chem. Lett. 5, 1421-1426 (2014).

10. Lien, D.-H. et al. Engineering light outcoupling in 2D materials. Nano Lett. 15, 1356-1361 (2015).

11. Lin, Y.-H. et al. Using $\mathrm{n}$-and $\mathrm{p}$-type $\mathrm{Bi}_{2} \mathrm{Te}_{3}$ topological insulator nanoparticles to enable controlled femtosecond mode-locking of fiber lasers. ACS Photonics 2, 481-490 (2015)

12. Haruyama, J. et al. Surface properties of $\mathrm{CH}_{3} \mathrm{NH}_{3} \mathrm{PbI}_{3}$ for perovskite solar cells. Acc. Chem. Res. 49, 554-561 (2016).

13. Murali, B. et al. The surface of hybrid perovskite crystals: a boon or bane. ACS Energy Lett. 2, 846-856 (2017).

14. Blancon, J.-C. et al. Extremely efficient internal exciton dissociation through edge states in layered 2D perovskites. Science 355, 1288-1292 (2017).

15. Tsai, M. L. et al. Single atomically sharp lateral monolayer $\mathrm{p}-\mathrm{n}$ heterojunction solar cells with extraordinarily high power conversion efficiency. Adv. Mater. 29, 1701168 (2017).

16. Kepenekian, M. et al. Concept of lattice mismatch and emergence of surface states in two-dimensional hybrid perovskite quantum wells. Nano Lett. 18 5603-5609 (2018).

17. Mercier, N. et al. Unique hydrogen bonding correlating with a reduced band gap and phase transition in the hybrid perovskites $\left(\mathrm{HO}\left(\mathrm{CH}_{2}\right) 2 \mathrm{NH}_{3}\right) 2 \mathrm{PbX}$ (X = I, Br). Inorg. Chem. 43, 8361-8366 (2004). 
18. Cheng, B. et al. Extremely reduced dielectric confinement in two-dimensional hybrid perovskites with large polar organics. Commun. Phys. 1, 80 (2018).

19. Guo, P. et al. Hyperbolic dispersion arising from anisotropic excitons in twodimensional perovskites. Phys. Rev. Lett. 121, 127401 (2018).

20. Peng, W. et al. Ultralow self-doping in two-dimensional hybrid perovskite single crystals. Nano Lett. 17, 4759-4767 (2017).

21. Heilmeier, G. H. \& Harrison, S. E. Implications of the intensity dependence of photoconductivity in metal-free phthalocyanine crystals. J. Appl. Phys. 34, 2732-2735 (1963).

22. Milot, R. L. et al. Charge-carrier dynamics in $2 \mathrm{D}$ hybrid metal-halide perovskites. Nano Lett. 16, 7001-7007 (2016).

23. Huang, J. et al. Understanding the physical properties of hybrid perovskites for photovoltaic applications. Nat. Rev. Mater. 2, 17042 (2017)

24. Blancon, J.-C. et al. Scaling law for excitons in 2D perovskite quantum wells. Nat. Commun. 9, 2254 (2018).

25. Schlegel, J. et al. Influence of carrier lifetime, transit time, and operation voltages on the photoresponse of visible-blind AlGaN metal-semiconductor-metal photodetectors. Jpn. J. Appl. Phys. 52, 08JF01 (2013).

26. Fang, H.-H. et al. Ultrahigh sensitivity of methylammonium lead tribromide perovskite single crystals to environmental gases. Sci. Adv. 2, e1600534 (2016).

27. $\mathrm{Hu}, \mathrm{L}$. et al. Investigation of the interaction between perovskite films with moisture via in situ electrical resistance measurement. ACS Appl. Mater. Interfaces 7, 25113-25120 (2015).

28. Ren, K. et al. Turning a disadvantage into an advantage: synthesizing highquality organometallic halide perovskite nanosheet arrays for humidity sensors. J. Mater. Chem. C. 5, 2504-2508 (2017).

29. Matis, B. R. et al. Surface doping and band gap tunability in hydrogenated graphene. ACS Nano 6, 17-22 (2012).

30. Leguy, A. M. et al. Reversible hydration of $\mathrm{CH}_{3} \mathrm{NH}_{3} \mathrm{PbI}_{3}$ in films, single crystals, and solar cells. Chem. Mater. 27, 3397-3407 (2015).

31. Chen, Z. \& Lu, Chi Humidity sensors: a review of materials and mechanisms. Sens. Lett. 3, 274-295 (2005)

32. Bi, H. et al. Ultrahigh humidity sensitivity of graphene oxide. Sci. Rep. 3, 2714 (2013).

\section{Acknowledgements}

We thank Xiaohe Miao for the help of X-ray diffraction measurement and data analysis. This work was financially supported by the King Abdullah University of Science and
Technology (KAUST) Office of Sponsored Research (OSR-2016-CRG5-3005), KAUST solar center (FCC/1/3079-08-01), and KAUST baseline funding.

\section{Author contributions}

B.C., T.-Y.L., and J.-H.H. conceived the idea. B.C. and T.-Y.L. synthesized the crystals, fabricated devices, and did the measurements. K.-T.H. and J.R.D.R. assisted the measurement. J.Y. and O.F.M. did the DFT calculation. B.C., T.-Y.L., P.-C.W., and J.-H.H. analyzed the data and wrote the paper. All the authors discussed the results and contributed to the manuscript.

\section{Additional information}

Supplementary Information accompanies this paper at https://doi.org/10.1038/s41467 018-07656-2.

Competing interests: The authors declare no competing interests.

Reprints and permission information is available online at http://npg.nature.com/ reprintsandpermissions/

Publisher's note: Springer Nature remains neutral with regard to jurisdictional claims in published maps and institutional affiliations.

(c) (i) Open Access This article is licensed under a Creative Commons Attribution 4.0 International License, which permits use, sharing, adaptation, distribution and reproduction in any medium or format, as long as you give appropriate credit to the original author(s) and the source, provide a link to the Creative Commons license, and indicate if changes were made. The images or other third party material in this article are included in the article's Creative Commons license, unless indicated otherwise in a credit line to the material. If material is not included in the article's Creative Commons license and your intended use is not permitted by statutory regulation or exceeds the permitted use, you will need to obtain permission directly from the copyright holder. To view a copy of this license, visit http://creativecommons.org/ licenses/by/4.0/.

(C) The Author(s) 2018 